XXIX International Conference on Photonic, Electronic, and Atomic Collisions (ICPEAC2015) IOP Publishing Journal of Physics: Conference Series 635 (2015) 092015

\title{
Dressing Effects in the Attosecond Transient Absorption Spectra of
}

\section{Doubly-Excited States in Helium}

\author{
L. Argenti*1, Á. Jiménez-Galán*, C. Marante*, C. Ott ${ }^{\dagger \ddagger}$, T. Pfeifer ${ }^{\dagger \ddagger}$, F. Martín* ${ }^{*} \S$ \\ * Departamento de Química, Módulo 13,Universidad Autónoma de Madrid, Cantoblanco 28049, Madrid, Spain \\ † Max-Planck Institut für Kernphysik, Saupfercheckweg 1, 69117 Heidelberg, Germany \\ ¥ Center for Quantum Dynamics, Ruprecht-Karls-Universität Heidelberg, 69120 Heidelberg, Germany \\ $\S$ Condensed Matter Physics Center (IFIMAC), Universidad Autónoma de Madrid, 28049 Madrid, Spain \\ ฯ IMDEA-Nanociencia, Cantoblanco, 28049 Madrid, Spain
}

Synopsis Strong-field manipulation of autoionizing states is a crucial aspect of electronic quantum control. Recent measurements of the attosecond transient absorption spectrum (ATAS) of helium dressed by a few-cycle visible pulse [C. Ott et al, Nature 516, 374 (2014)] provide evidence of the inversion of Fano profiles. With the support of accurate $a b$-initio calculations that reproduce the results of the latter experiment, here we investigate the new physics that arise from ATAS when the laser intensity is increased. In particular, we show that (i) previously unnoticed signatures of the dark $2 p^{2}{ }^{1} \mathrm{~S}$ doubly excited state are observed in the experimental spectrum, (ii) inversion of Fano profiles is predicted to be periodic in the laser intensity, and (iii) the ac-Stark shift of the higher terms in the $s p_{2, n}^{+}$autoionizing series exceeds the ponderomotive energy, which is the result of a genuine two-electron contribution to the polarization of the excited atom.

ATAS [1] has affirmed itself as a valid technique to monitor and control electronic wavepackets [1-3]. Control is achieved with a laser pulse that imparts an ac-Stark shift to the wavepacket components, thus altering their relative phases. The latter map onto the asymmetry of their resonant profiles in the ATAS spectrum [4]. This principle has been recently applied to a two-electron wave packet in the helium atom, formed by the $s p_{2, n}^{+}{ }^{1} \mathrm{P}^{o}$ doubly excited states [3]. The experiment evidenced the Autler-Townes splitting of the $s p_{2,2}^{+}$state, which is coupled to the $2 p^{2}{ }^{1} \mathrm{~S}$ state, as well as inversion of the Fano profile in the higher terms of the $s p_{2, n}^{+}$series as a function of both the time delay between the XUV and the VIS pulses, and the intensity of the VIS dressing field [4].

Here we show, by means of accurate ab-initio calculations $[3,5,6]$ that reproduce the experimental results for the helium atom, that ATAS can be used to measure the non-perturbative response of autoionizing states to external dressing fields. In particular, we show that the AutlerTownes splitting of the dressed $2 p^{2}{ }^{1} \mathrm{~S}$ "dark" state can be measured, and that the asymmetric resonant features in the spectrum undergo repeated inversions as the intensity of the driving laser is increased. The ac-Stark shift of the doubly-excited states, which are important parameters for the design of quantum-control protocols, increases non-linearly with the intensity of the dressing field; for the higher states of the autoionizing $s p_{2, n}^{+}$series, it exceeds by more than twice the theoretical single-active-electron limit. Finally, we establish a relationship between the ATAS spectrum and the non-diagonal components of the XUV electrical susceptibility, thus extending multidimensional spectroscopy to the XUV domain (Fig. 1), which provides an alternative way to measure shifts of and coherences between states above the ionization threshold.

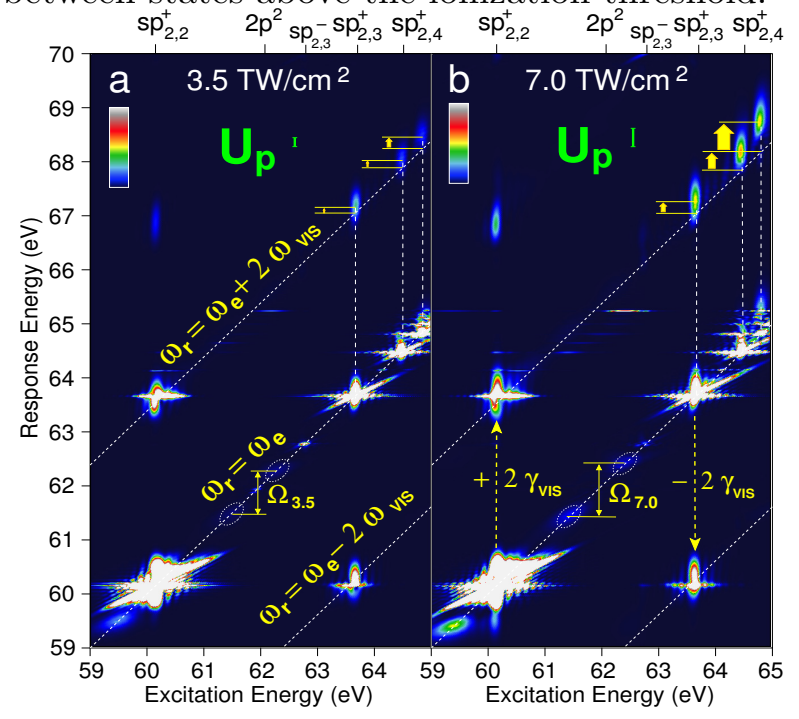

Figure 1. Reconstruction of the non-diagonal XUV electrical susceptibility $\left|\chi_{\text {nd }}\left(\omega_{\mathrm{r}}, \omega_{\mathrm{e}}\right)\right|^{2}$ of dressed helium.

\section{References}

[1] E. Goulielmakis et al. 2010 Nature 466, 739.

[2] A. R. Beck et al. 2014 New J. Phys. 16, 113016.

[3] C. Ott et al. 2014 Nature 516, 374.

[4] C. Ott et al. 2013 Science 516, 374.

[5] L. Argenti and E. Lindroth 2010 PRL 105, 053002.

[6] L. Argenti et al. 2015 submitted.

\footnotetext{
${ }^{1}$ E-mail: luca.argenti@uam.es
} 